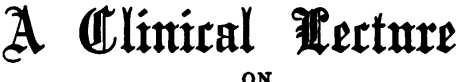

\section{CANCER OF THE PANCREAS.}

Delivered at St. Bartholomew's Hospital,

BY SIR LAUDER BRUNTON, M.D., F.R.S.

There are two cases to which I wish to draw your attention to-day because they are both of extreme interest, and they both presented the common features of jaundice, pain, and emaciation.

Case 1.

The first case was that of a woman aged 44. When admitted she complained of pain in the chest and back and abdomen. Five months prior to admission she had been suffering from dyspepsia, and three weeks before admission she took to her bed for weakness, pain in the back, and sickness in the morning. When admitted she was very pale, there was a slight systolic murmur over the pulmonary artery and another limited one over the left nipple. Both of those seemed to be simply anaemic, very soft and of limited distribution. On examining the abdomen the stomach was not found to be dilated because, although you might almost imagine from the drawing which I have made, and in which you see the stomach reaching nearly to the umbilicus, that it was dilated, that is not so. I have made many hundred observations on the position of the lower margin of the stomach in women and have found that in the great majority it reaches down to the um. bilicus. So I should regard this as the normal position of the great curvature of the stomach in women, although in men such a position would indicate a very considerable amount of dilatation. On palpation distinct resistance was felt a little above the umbilicus in the middle line. I was uncertain at the time whether this was due to some tumour in the stomach itself or simply to the hardness of the recti contracting so as to shield a tender spot in the stomach below. But Mr. Von Bergen was more fortunate, and he was able to find a distinct nodule in the stomach. As I have had occasion to show you before, the pylorus varies very much in its position from time to time, and a pyloric tumour may be felt in almost any part of the epigastric or in the umbilical regions, or on either side of the middle line as far as the ribs on the left side. Some days afterwards this swelling had not only changed its character but it was felt much more distinctly to the right. Those symptoms I have described led one naturally to the diagnosis of cancer of the stomach. But after a little while longer the patient began to get janndice and the jaundice became very deep and the emaciation exceedingly rapid, so much so that I was inclined to think we had to deal here not with a case of simple carcinoma of the stomach but one in which cancer of the pancreas was superadded. The emaciation was progressive and rapid, and finally the patient died. On post-mortem examination cancer was found in the stomach, forming a large ulcer near the pyloric orifice with somewhat thickened edges but without any great thickening. The floor of the ulcer was smooth, and then came the explanalion of the intense jaundice which the patient presented. The glands about the pancreas and in the hilum of the liver were invaded by new growth, forming a very firm mass. The pancreas itself was not involved.
The fact was that the mass of glands invaded by cancer was firmly pressed against the pancreas and had no doubt caused obstruction of the common bile duct; whether it entirely prevented the flow of pancreatic juice into the bowel I casınot say, but I am inclined to think that it noust have done so to a great extent judging from the very rapid emariation of the patient. Here, then, we have to deal with a case of cancer of the stomach in which the glands were so much involved secondarily as to bring about the symptoms of pancreatic cancer, although the pancreas itself was absolutely free from the disease.

\section{Case ir.}

The next case is one of cancer of the pancreas. H. B., aged 54 a porter, admitted complaining of pain in the abromen. Twelve weeks before admission he had pain in the epigastrium and in the back, with a feeling of weight aft. $r$ his meals. Two days after the rommencement of this pain his eyes became zellow, and in three more dajs his skin was yellow. He noticed also that hin motions were light coloured. He had no vomiting and no diarrhuea. When the patient was admitted he looked thin and rather emaciated. H1s chest was somewhat barrel-shaped and the costal angle was very wide. There was accentuation of the second sound over the aortic orifice. The apex beat was in the normal position, the first sound was slightly prolonged, but there was nothing amounting to a real murmur. The liver was very large, and the edge of it was quite smooth. But the feature which most attracted one's attention was that under the ribs, on the right side, was a rounded boss circular, in outline. It was not tender on pressure and there was no rise of temperature, the patient's temperature being normal. That seemed to dispose of the idea that we might have here a case of abscess in the liver. The surface of the liver was so smooth that at first the thought occurred to me that although the general appearance of the patient suggested malignant disease, it might possibly be a case of hypertrophic cirrhosis. But a few days afterwards a projection was found from the lower part of the liver, which was hard and triangular ; indeed, it felt exactly like a nodule of malignant disease. Then 1 had no doubt that the liver was affected by cancer. A few days after this the patient passed a dark tarry motion, and the pain continued very severe, and was only alleviated by the use of morphine. He lost strength rapidly and then had a one-sided convulsion and died about a fortnight later, his temperature for the two days before death having risen to $100^{\circ} \mathrm{F}$. and $10{ }^{\circ} \mathrm{F}$

There is very little doubt that the convulsion was due to a uraemic condition; the kidneys were not excreting the waste products of metabolism.

The Diagnosis of Pancreatic Cancer.

Now, in a paper written by Dr. Herringham, and published in the St. Bartholomew's Hospital Reports, vol. xxx, 1894, he lays down these conclusions

That when a patient who suffers from deeply-seated pain in the epigastric or the hepatic region, along witb progressive emaciation, but without signs definitely indicating gastric cancer, has jaundice and dilatation of the gall bladder without a history of biliary colic, by far the most probable diagnosis is that he has primary cancer of the pancreas.

Here then we have the symptoms indicated by Dr. Herringham: (1) The deeply-seated pain, which in this case was very severe; (2) the progressive emaciation; (3) the absence of signs indicating gastric cancer, there having been 
no vomiting in this case; and $\left(_{4}\right)$ the jaundice occurring without a history of biliary colic. Those are the symptoms. In looking up the subjective signs as given by Fitz in Clifford Allbutt's System of Medicine, I find that he says that the "most characteristic sign of cancer of the pancreas is a tumour in the epigastric or umbilical region, which is to be observed as a deep-seated rounded or elongated, sometimes nodulated, swelling varying in density and defined with difficulty."

Now we had here, as a very striking feature of the case, a tumour in the epigastric region, but it differed from the tumour as described by Fitz in several respects. It seemed to be higher up than one would expect any tumour of th pancreas to be, and instead of being deeply seated it seemed to be quite superficial. What we felt was almost certainly not the pancreas itself but the liver, which was raised over a large mass of pancreatic cancer. The specimen is here. The pancreas was infiltrated with new growth throughout its whole structure, part of it was soft and partwas hard, and the hard part at the head of the pancreas had pressed upon the bile ducts so as to completely obstruct their course, and, indeed, the bile duct seemed to pass into the mass. You will notice that the liver is deeply stained with bile, and the ducts are much distended throughout the organ. At the necropsy these ducts, on the slightest pressure, exuded a very thick and very dark bile, almost like tar, and the ducts, for some considerable distance up, were very largely dilated. You see that the liver itself seems to be free from infiltration by new growth, and the new nodule that we felt really appears to have been the enlarged gall bladder which was extremely tense, and projected beyoud the edge of the liver. It was so tense that it gave one's fingers the feeling of not being a soft elastic structure such as the gall bladder usually feels to be, but to be a hard nodular growth. The other organs were very fairly. healthy. The mitral valves were slightly adherent together, and to this was no doubt due the somewhat prolonged first sound. This specimen is particularly interesting in regard to the methods of diagnosis which have been proposed by Fitz, because it dismisses a number of things that have been supposed to be methods of ascertaining the presence of cancer of the pancreas. For example, it has been supposed that in cancer of the pancreas you would find glucose in the urine. Such was not the case here. But Fitz has proposed, as the surest way of ascertaining the presence of cancer of the pancreas, alterations either in the food or physie introduced into the intestinal canal, and he mentions that in cases of "cancer of the pancreas, if the patient be fed upon meat, large quantities of undigested muscular fibre are to be found in the stools, the reason being that if the pancreatic juice does not enter the intestine the muscular fibre will remain undigested. Another method that he proposed is for salol to be given in doses of one drachm per diem in divided portions. The effect of the pancreatic juice upon this drug, which is a salicylate of phenyl, is to split it up, forming salicylic acid and phenol. The phenol is excreted in the urine, and there its presence can be ascertained by bromine water. Now, these tests were not applied in this case, and it is a pity they were not, but if we had applied them we should in all probability have come to a wrong diagnosis, because, oddly enough, although the hard nodulein the head of the pancreas presses upon the gall ducts, and these are completely prevented from emptying themselves yet a duct of the pancreas remains free (cf. Fig. 1) and some pancreatic juice entered the intestine, for you see here a certain amount of post-mortem digestion by the pancreas. In this case, then, if we had applied those tests we should have found evidence of the presence of pancreatic juice in the intestine, and we should have come to the conclusion that there was in all probability cancer of the liver but no cancer of the pancreas. So that at present you see that the tests which were thought to be reliable cannot be implicitly trusted, and we must fall back upon the symptoms that $I$ have mentioned to you before-deepseated pain, progressive emaciation, jaundice, and distension of the gall bladder. The round swelling may or may not be there, and the presence or absence of sugar in the urine does not help very much. But excessive pain in this case was a very marked symptom, and the difficulty in such cases is to distinguish between the pain due to pancreatic disease and pain due to perihepatitis. Of course in perihepatitis you may hear a friction if the part of the liver affected happens to be under the abdominal wall; but if the npper surface of the posterior surface of the liver be affected with perihepatitis there may be a great deal of pain and yet no friction. The difficulty is so great in distinguishing between the pain due to perihepatitis and cancer of the liver and pain due to cancer of the pancreas, that I suppose we have practically to fall back to a great extent upon the very rapid emaciation. In cases of cancer of the liver without cancer of the pancreas the malignant disease may be present for many months together. sometimes I believe for years together. But in cancer of the pancreas the course of the symptoms is very rapid, and you will have noticed that the present one only lasted four months from the first symptom to the fatal termination.

As far as treatment is concerned, medicines are of little good except in so far as you are able by them to relieve the pain by sedatives, such as morphine. Uperation, as you will have seen, would be out of the question.

I have brought those cases before you because, meeting a medical colleague a little while ago, he seemed to doubt whether cancer of the pancreas could be, with any degree of certainty, diagnosed during life. I think that although these cases show how some of the signs upon which the greatest amount of reliance can be placed are not trustworthy, yet by attending to those rules which Dr. Herringham laid down, we may come to a fairly accurate diagnosis. Still the case which I have put before you of cancer of the stomach shows you must be on your guard against assuming the presence of cancer in the pancreas if you have already got indications of cancer in the stomach, because the glands there may become so much affected as to simulate the presence of cancer of the pancreas.

[NOTE--Since this lecture was delivered a new met hod of examining the urine, which seems likely to be of great service in the diagnosis of cancer of the pancreas, has been described by Cammidge, Lancet, March 19th, 1904, p. 782, and Abstract in BRITISH MEDICAL JOURNAL, April 2nd, 1904 p. $776 . j$

\section{An Autregs \\ ON}

\section{THE MODERN PURSUIT OF NOVELTIES} IN MEDICINE.

Delivered before the Reigate Division of the South-Eastern Branch of the British Medical Association at Dorking.

By Sir DYCE DUCKWORTH, M.D., LL.D., F.R.C.P., Physician and Lecturer on Clinical Medicine, St. Bartholomew's Hospital.

Gentlemen, - You are aware that the changes in medical education which have gradually come about in the last half of this century are numerous, and some of them so radical that they amount to a complete transformation of the student's curriculum. There are many reasons for these changes. Not the least of them is the general and astounding advance in all the sciences, and in those especially on which the art of medicine is based, by which I mean those of biology.

I hope you have no misunderstanding with respect to the position of medicine itself. It never was, and it never will be, a science; yet it is scientific, and its foundations lie, and must ever lie, on the bedrock of many sciences. Medicine, as physicians understand it, is an art, and all good physicians are good artists. It is possible to be a great medical scientist, and an inferior medical artist. We may hold that true physicians, like true poets, are born and not made, if we safeguard the assertion by adding that their best qualities do not come by instinct, ready-made, but are reached only by assiduous study and unceasing accurate observation. There is no royal road to their attainments, however keen their wits or precise their logic. The mental temper which alone befits the pure scientist would never quite become the investigator of disease, or the student of clinical problems, and for this reason, that the latter has to act promptly and make the best of the facts before him, often without attaining the certainty and exactness of the requirements of the physicist. The difficulties and abstruseness in the particular case before the physician may never be entirely cleared up, and these constitute distressing factors which sadly exercise the purely scientific mind and tend to avert it from the practice of our art. This has indeed often happened, and men who have eagerly taken up our work have found themselves unable to combat with the oft-recurring uncertainties which are inevitable in practice. They have gone off into other fields of research for which their mental qualities were better fitted. We cannot afford to sit down and bewail our inabilities, or shrink from the alluring problems which await us. For what is the reason of our existence in the body politic? Is it not 